\title{
EDITORIAL
}

\section{Growing collaborations between Chinese and UK young scholars on chemical science and technology}

\author{
Weiping Wu $(\bowtie)^{1}$, Xiaolei Fan $(\bowtie)^{2}$, Yongliang Li $(\bowtie)^{3}$, Ruijiao Dong $(\bowtie)^{4}$ \\ 1 Shanghai Institute of Optics and Fine Mechanics, Chinese Academy of Sciences, Shanghai 201800, China \\ 2 Department of Chemical Engineering and Analytical Science, School of Engineering, The University of Manchester, Manchester, M13 9PL, UK \\ 3 Birmingham Centre for Energy Storage, School of Chemical Engineering, University of Birmingham, Birmingham, B15 2TT, UK \\ 4 Shanghai Center for Systems Biomedicine, Key Laboratory of Systems Biomedicine (Ministry of Education), Shanghai Jiao Tong University, \\ Shanghai 200240, China
}

(C) Higher Education Press 2020

Following the CSCST-25 Special Issue [1] we continued this issue based on the $26^{\text {th }}$ CSCST-SCI annual conference (CSCST-SCI 26), which was held at the Society of Chemical Industry (SCI) headquarter, 14/15 Belgrave Square, London SW1X 8PS on the $4^{\text {th }}$ and $5^{\text {th }}$ September 2019. The annual conference had 4 sessions with 20 oral and 14 poster presentations, which featured several plenary and keynote lectures by national and international eminent speakers. International researchers and delegates from China and Australia, and members of other $\mathrm{SCl}$ groups (SCl Energy Group and SCI London Regional Group) also joined the two-day event. Dr. Weiping Wu, Chairman of SCl Chinese UK Group introduced the history, membership, activities of SCl and SCI Chinese UK Group [2]. Mr. Sunan Jiang, Minister Counsellor for Science and Technology Affairs, Embassy of the People's Republic of China in the United Kingdom gave a talk on the China-UK collaborations in science and innovations. Sharron Todd, Chief Executive Officer of $\mathrm{SCl}$ warmly welcomed everyone to the SCl headquarter and the conference, she awarded prizes to the oral presentation winners of CSCST-SCI 26.

The theme of the conference is Energy and Environment, two important areas of Chemical Engineering. This issue supports the community and rapidly reports the recent exciting discoveries in this field. All manuscripts were peerreviewed, and finally 19 contributions were accepted for publication in the Special Issue. The contributions cover a wide spectrum of research activities of the community including novel materials for solar cells [3,4], solar thermal energy harvest/storage [5,6], photocatalysis and electrocatalysis [7,8], hydrogen production and fuel cells $[9,10]$, sustainable materials $[11,12]$ and advanced chemical engineering processing $[13,14]$. The four review articles present the bio-oil upgrading in supercritical fluids [15], the computational design of heterogeneous catalysts [16], the state-of-the-art of polymer based dielectrics for high-energy storage [17] and advanced chemical processing and photothermal materials for solar-powered steam generation [18].

Based on the aesthetic appeal, one cover artwork was selected by the editors. The cover article is from the paper of Montazersadgh et al. on the electrolytic cell engineering and device optimization for electrosynthesis of e-biofuels via co-valorisation of bio-feedstocks and captured $\mathrm{CO}_{2}$ [8]. The authors presented a numerical model of a continuous-flow design considering various $\mathrm{CO}_{2}$ anodic and cathodic reactions produce fuels (e.g., methanol) and high value-added chemicals (e.g., selective oxidation of alcohols, aldehydes, carboxylic acids and amines/amides), to determine the most techno-economically feasible configurations from the aspects of energy efficiency, environmental impact and economical values.

Received October 28, 2020

E-mails: wuwp@siom.ac.cn (Wu W); xiaolei.fan@manchester.ac.uk (Fan X); y.li.1@bham.ac.uk (Li Y); drj021@sjtu.edu.cn (Dong R) 


\section{References}

1. Fan X L, Jia W W. Preface to the CSCST-25 Special Issue. Frontiers of Chemical Science and Engineering, 2019, 13(4): 629-631

2. Chinese UK International Groups. Society of Chemical Industry Website, 2020

3. Wang Y, Yang L, Dall'Agnese C, Chen G, Li A, Wang X. Spray-coated $\mathrm{SnO}_{2}$ electron transport layer with high uniformity for planar perovskite solar cells. Frontiers of Chemical Science and Engineering, 2021, 15(1): 180-186

4. Liu L, Zhang H, Xiao B, Liu Y, Xu B, Wang C, Wen S, Zhou E, Chen G, Im C, Tian W. Effects of BTA 2 as the third component on the charge carrier generation and recombination behavior of $\mathrm{PTB}_{7}: \mathrm{PC}_{71} \mathrm{BM}$ photovoltaic system. Frontiers of Chemical Science and Engineering, 2021,15 (1): $127-137$

5. Liu F, Lai Y, Zhao B, Bradley R, Wu W. Photothermal materials for efficient solar powered steam generation. Frontiers of Chemical Science and Engineering, 2019, 13(4): 636-653

6. Suleiman B, Yu Q, Ding Y, Li Y. Fabrication of form stable $\mathrm{NaCl}-\mathrm{Al}_{2} \mathrm{O}_{3}$ composite for thermal energy storage by cold sintering process. Frontiers of Chemical Science and Engineering, 2019, 13(4): 727-735

7. Lu J, Lan L, Liu X, Wang N, Fan X. Plasmonic Au nanoparticles supported on both sides of $\mathrm{TiO}_{2}$ hollow spheres for maximising photocatalytic activity under visible light. Frontiers of Chemical Science and Engineering, 2019, 13(4): 665-671

8. Montazersadgh F, Zhang H, Alkayal A, Buckley B, Kolosz B, Xu B, Xuan J. Electrolytic cell engineering and device optimization for electrosynthesis of e-biofuels via co-valorisation of bio-feedstocks and captured $\mathrm{CO}_{2}$. Frontiers of Chemical Science and Engineering, 2021,15 (1): 208-219

9. Ismaila A, Chen X, Gao X, Fan X.Thermodynamic analysis of steam reforming of glycerol (srg) for hydrogen production at atmospheric pressure. Frontiers of Chemical Science and Engineering, 2021, 15(1): 60-71

10. Zhang H, Liang J, Xia B, Li Y, Du S. Ionic liquid modified Pt/C electrocatalysts for cathode application in proton exchange membrane fuel cells. Frontiers of Chemical Science and Engineering, 2019, 13(4): 695-701

11. Yan Y, Huang P, Zhang H. Preparation and characterization of novel carbon molecular sieve membrane/PSSF composite by pyrolysis method for toluene adsorption. Frontiers of Chemical Science and Engineering, 2019, 13(4): 772-783

12. Jiang S, Daly H, Xiang H, Yan Y, Zhang H, Hardacre C, Fan X. Microwave-assisted catalyst-free hydrolysis of fibrous cellulose for deriving sugars and biochemicals. Frontiers of Chemical Science and Engineering, 2019, 13(4): 718-726

13. Yu T, Cheng J, Li L, Sun B, Bao X, Zhang H. Current understanding and applications of the cold sintering process. Frontiers of Chemical Science and Engineering, 2019, 13(4): 654-664

14. Gao X, Shu D, Li X, Li H. Improved film evaporator for mechanistic understanding of microwave-induced separation process. Frontiers of Chemical Science and Engineering, 2019, 13(4): 759-771

15. Omar S, Yang Y, Wang J. A review on catalytic \& non-catalytic bio-oil upgrading in supercritical fluids. Frontiers of Chemical Science and Engineering, 2021, 15(1): 4-17

16. Shi H, Zhou T. Computational design of heterogeneous catalysts and gas separation materials for advanced chemical processing. Frontiers of Chemical Science and Engineering, 2021, 15(1): 49-59

17. Sun W, Mao J, Wang S, Zhang L, Cheng Y. Review of recent advances of polymer based dielectrics for high-energy storage in electronic power devices from the perspective of target applications. Frontiers of Chemical Science and Engineering, 2021, 15(1): 18-34

18. Zhang T, Wang S, Zhang X, Fu M, Yang Y, Chen W, Su D. Recent progress on nanostructure-based broadband absorbers and their solar energy thermal utilization. Frontiers of Chemical Science and Engineering, 2021, 15(1): 35-48

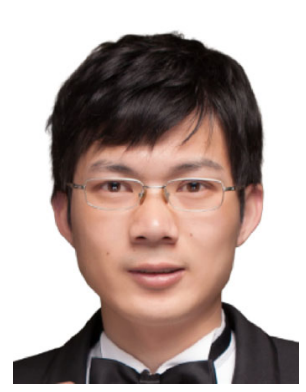

Dr. Weiping Wu is the professor at Shanghai Institute of Optics and Fine Mechanics (SIOM), Chinese Academy of Sciences (CAS). He graduated with a bachelor's degree in material science and engineering at Shanghai Jiao Tong University in 2004. He received his Ph.D. in chemistry from the Insitute of Chemistry, Chinese Academy of Sciences (ICCAS) in 2010. From 2010 to 2013 he worked as a research associate at the University of Cambridge. He was a research fellow at the Optoelectronics Research Centre (ORC), the University of Southampton from 2013 to 2015. He took the George Daniels Lecturership at City, University of London from September 2015 to September 2020. He is the chairman of Chinese UK Group of Society of Chemical Industry (SCI), the young observer of the International Union of Pure and Applied Chemistry (IUPAC) $47^{\text {th }}$ Congress, $50^{\text {th }}$ General Assembly and Centenary, Paris in 2019. He is a member of the Royal Society of Chemistry (RSC), and a senior member of OSA (The Optical Society) since 2020 . 


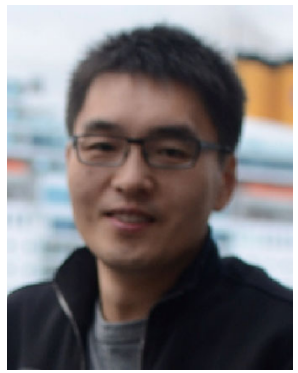

Dr. Xiaolei Fan is a reader in chemical engineering at The University of Manchester. Fan received his Ph.D. in chemical engineering from the University of Bath in 2010. Fan is the current chair of CSCST-UK, and his research broadly encompasses the heterogeneous catalysis, catalytic reaction engineering, process intensification and porous materials.

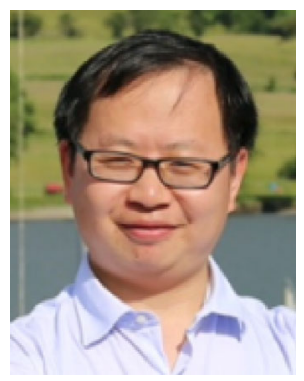

Dr. Yongliang $\mathrm{Li}$ is a reader in thermal energy engineering in the School of Chemical Engineering at the University of Birmingham. He received his Ph.D. in chemical and process engineering from the University of Leeds in 2011. His research interests are energy conversion, storage, and management. He has been receiving funding from the EPSRC, EU, Innovate UK, Royal Society, British Council, and major industrial companies and has published about 100 peer-reviewed journal articles and book chapters.

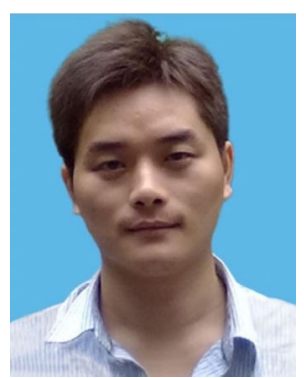

Dr. Ruijiao Dong is an associate professor in the Shanghai Center for Systems Biomedicine at Shanghai Jiao Tong University. He completed his B.Sc. degree in materials science at Wuhan Textile University and his M.Sc. degree in polymer chemistry at Donghua University. In 2009, he joined Prof. Xinyuan Zhu's group as a Ph.D. student in the Department of Chemistry at Shanghai Jiao Tong University. From 2015 to 2020, he worked as a research associate at Imperial College London. His current research interests focus on the synthesis and development of precision drug/ gene delivery vehicles and sequence-controlled polymeric materials.

Wu, Fan, Li and Dong are the invited Guest Editors of Frontiers of Chemical Science and Engineering. 